\title{
SENSITIVITY WITH RESPECT TO THE YIELD CURVE: DURATION IN A STOCHASTIC SETTING
}

\author{
PAUL C. KETTLER, FRANK PROSKE, AND MARK RUBTSOV
}

\begin{abstract}
Bond duration in its basic deterministic form is a concept well understood. Its meaning in the context of a yield curve on a stochastic path is less well developed. We extend the basic idea to a stochastic setting. More precisely, we introduce the concept of stochastic duration as a Malliavin derivative in the direction of a stochastic yield surface modeled by the Musiela equation. Further, using this concept we also propose a mathematical framework for the construction of immunization strategies (or delta hedges) of portfolios of interest-ratesensitive securities with respect to the fluctuation of the whole yield surface.
\end{abstract}

\section{INTRODUCTION}

The concept of bond duration dates to a foundational book defining the idea (Macaulay 1938). Through the years there have been many presentations on the idea. One of note is (Jarrow 1978). Other tracts obtain, most frequently addressing the bond with periodic coupons and a terminal payment of principal. Such discussions tend to concentrate on the idea of an annuity as the sum of a geometric series, presented in a variety of flavors. We eschew these notions as being of scant academic interest, and focus on the continuously compounded zero coupon bond as a building block, leaving the construction of instruments with component payments to others. See Appendix A for a brief discussion of Macaulay duration in context.

The bond market worldwide has about $\$ 45$ trillion outstanding, with about $\$ 1$ trillion trading on a typical day. Other than price and yield, the most widely quoted parameter in the market, without question, is duration. It appears on quotation screens, on traders' lips, and in all manner of literature on the market. Yet the concept, which addresses the sensitivity of a bond's price with respect to changes in yield, assumes a uniform rate of interest through the life of a bond, an unrealistic hypothesis.

In basic bond analysis one considers a zero coupon bond with present value (or price) $v$ given as a function of a level interest rate $r$, maturing to future value $\$ 1$ at time $T$. The relationship of variables is this.

$$
v=\mathrm{e}^{-r T}
$$

Date: 10 May 2010.

2010 Mathematics Subject Classification. Primary: 60G07, 60G60. Secondary: 60H05, 60H07. 1991 Journal of Economic Literature Subject Classification. C41, C51.

Key words and phrases. Duration; Fréchet derivative; stochastic process; function state space; Gaussian random field; Malliavin calculus; backward stochastic differential equation.

This version of the paper was compiled in $\mathcal{A} \mathcal{M} \mathcal{S}$-IATEX with Palatino and Euler Virtual Math fonts [both by Hermann Zapf], and University of Chicago Press bibliography and citation [author-date] style. 
The quantity

$$
d:=\frac{1}{v} \frac{\partial v}{\partial r}=\frac{\partial}{\partial r} \log v=-T
$$

is known as the duration, and the quantity

$$
c:=\frac{1}{2 v} \frac{\partial^{2} v}{\partial r^{2}}=\frac{1}{2} T^{2}
$$

is known as the convexity. Note that $d$ and $c$ are the coefficients, respectively, of $r$ and $r^{2}$ in the Taylor series expansion of $v$.

$$
v=1-\operatorname{Tr}+\frac{1}{2} T^{2} r^{2}-\ldots
$$

Bond traders routinely employ duration and convexity in market analysis to estimate the effects of rate changes.

An important fact about duration, which makes it useful for portfolio analysis, is that the duration of a portfolio is the average of the component durations weighted by present values. A two security case is sufficient to illustrate. Let

$$
v=\alpha_{1} v_{1}+\alpha_{2} v_{2}=\alpha_{1} \exp \left(-r T_{1}\right)+\alpha_{2} \exp \left(-r T_{2}\right)
$$

Then

$$
d=-\frac{\alpha_{1} v_{1}}{\alpha_{1} v_{1}+\alpha_{2} v_{2}} T_{1}-\frac{\alpha_{2} v_{2}}{\alpha_{1} v_{1}+\alpha_{2} v_{2}} T_{2}
$$

One may generalize this concept of bond to incorporate a piecewise constant interest rate $r(s)$, where

$$
r(s)=\left\{\begin{array}{llr}
r_{1} & , \text { if } & 0=: s_{0} \leq s<s_{1} \\
r_{2} & , \text { if } & s_{1} \leq s<s_{2} \\
\cdots & & \\
r_{n} & , \text { if } & s_{n-1} \leq s \leq s_{n}:=T
\end{array}\right.
$$

Then Equation (1.1) becomes

$$
v=\exp \left[-\sum_{i=1}^{n} r_{i}\left(s_{i}-s_{i-1}\right)\right]
$$

From this expression we obtain the $i^{\text {th }}$ partial duration

$$
d_{i}:=\frac{\partial}{\partial r_{i}} \log v=-\left(s_{i}-s_{i-1}\right) \quad, 1 \leq i \leq n
$$

and the $i^{\text {th }}$ partial convexity

$$
c_{i}:=\frac{1}{2}\left(s_{i}-s_{i-1}\right)^{2} \quad, 1 \leq i \leq n
$$

Observe that the partial durations add to the total duration, whereas the partial convexities (and higher order related partial terms) do not. 
One may elaborate further on the themes of Equations (1.1) and (1.3) by putting $r$ and the $\left\{r_{i}\right\}$ on stochastic paths. To start, denote by $P(t, T)$ the price at time $t$ of a zero coupon bond, which pays $\$ 1$ at maturity $T$. Then one can define instantaneous forward rates as

$$
f(t ; T)=-\frac{\partial \log (P(t, T))}{\partial T}, \quad 0 \leq t \leq T
$$

for each maturity T. See (Heath, Jarrow, and Morton 1992). So we can recast Equation (1.1) as

$$
v=P(t, T)=\exp \left(-\int_{t}^{T} f(t, s) \mathrm{d} s\right)
$$

Since the outcome of future interest rates is not known in advance it is reasonable to model instantaneous forward rates $\{f(t, s)\}_{0 \leq s \leq t}$ as stochastic processes. In this context we may interpret $f(t, s)$ as the overnight interest rate at (future) time $t$ as seen from time $s$. The case $f(t, t)=: r(t)$ is simply the overnight rate, or short rate.

The literature is replete with examples on interest rates. A small sample of papers, not otherwise cited in the text, is this (Vašíček 1977; Rendleman and Bartter 1980; Cox, Ingersoll, Jr., and Ross 1985; Lee and Ho 1986; Black, Derman, and Toy 1990; Ritchken and Sankarasubramanian 1995; Musiela 1995; Chen 1996a; Chen 1996b; Björk, Christensen, and Gombani 1998; Björk and Gombani 1999; Vargiolu 1999; Filipović and Zabczyk 2002; Aihara and Bagchi 2005; Filipović and Tappe 2008). All address stochastic interest rates in financial modeling. Of interest within are these references including co-author Marek Musiela: (Brace and Musiela 1994; Brace, Gątarek, and Musiela 1997; Musiela and Rutkowski 1997; Goldys, Musiela, and Sondermann 2000).

As mentioned above the classical duration is based on the assumption that interest rates are flat or piecewise flat. This assumption is quite unrealistic and only applies to sensitivity measurements with respect to parallel shifts of interest rates. The latter is especially unsatisfying for a trader who manages a complex portfolio of interest-rate-sensitive securities (as, e.g., caps, swaps, bond options, etc.) In this case it would be desirable to measure the interest rate risk of the portfolio with respect to the stochastic fluctuations of the whole term structure or even the yield surface, that is

$$
(t, x) \longmapsto Y(t, t+x),
$$

where $Y(t, T)$ is the yield given by

$$
Y(t, T)=-\frac{1}{T-t} \log P(t, T)
$$

Here $x$ in Equation (1.6) stands for the time-to-maturity.

Using the relation of Equation (1.5) we can represent the yield surface $Y_{t}(x):=Y(t, t+x)$ as

$$
Y_{t}(x)=\frac{1}{x} \int_{0}^{x} f_{t}(s) \mathrm{d} s,
$$

where $f_{t}(s):=f(t, t+s)$. Because of the linear correspondence of Equation (1.7) between the yield curves $Y_{t}(\cdot)$ and the forward curves $f_{t}(\cdot)$ we can and will refer to

$$
(t, x) \longmapsto f_{t}(x)
$$


as the yield surface in this paper.

Assuming, e.g., the Heath-Jarrow-Morton [HJM] model for the dynamics of instantaneous interest rates, one shows under certain conditions that the yield surface of Mapping (1.8) is described by a stochastic partial differential equation [SPDE], called the Musiela equation. See (Heath, Jarrow, and Morton 1992; Goldys, Musiela, and Sondermann 2000).

In this paper we wish to develop an analogous concept to the classical duration of Macaulay in the HJM setting. More precisely, we want to measure the sensitivity of interest rate claims with respect to the Musiela dynamics of the yield surface, as in Equation (1.8).

An apparently analogous way to the classical case would be to define the duration of an interest-rate security by means of the Fréchet derivative for each interest rate scenario. However, interest rate securities, or even dynamically hedged portfolios composed of them, are in general complicated functionals of the yield surface, and are usually not even continuous.

In order to overcome this problem one may think of weaker forms of derivatives than the Fréchet derivative to measure interest rate sensitivities. A possible candidate could be the Malliavin derivative. This derivative, which is treated in Section 2, can be considered as a stochastic Gateaux derivative.

In this paper we want to base the stochastic duration concept on this stochastic Gateaux derivative. This concept is analogous to the classical one in the sense that it relies on the derivative of an infinite-dimensional version of the Taylor expansion as in Equation (1.2). Using this concept we also define stochastic convexity as a measure for the "curvature" of yield surface movements.

The paper is organized as follows: In Section 2 we define the concept of stochastic duration by using Malliavin calculus for general Gaussian random fields. In Section 3 we propose a mathematical framework for the construction of immunization strategies of portfolios, which are composed of interest rate instruments.

\section{An EXPANDED CONCEPT OF DURATION Via MaLliaVin CALCUlus}

In this section we want to elaborate a duration concept for stochastic yield curves. This definition extends the classical duration of Macaulay to a stochastic setting.

Denote by $P(t, T)$ the price at time $t$ of a zero coupon bond, which pays $\$ 1$ at maturity $T$. Suppose that the bond prices are modeled by non-negative adapted processes $\left\{P(t, T\}_{0 \leq t \leq T}\right.$ for each $T>0$ on a filtered probability space

$$
\left(\Omega, \mathcal{F},\left\{\mathcal{F}_{t}\right\}_{t \geq 0}, P\right)
$$

In the following we assume that the bond prices $P(t, T)$ are described by the HJM model (Heath, Jarrow, and Morton 1992); that is, the bond prices take the form

$$
P(t, T)=\exp \left(-\int_{0}^{T} f(t, s) \mathrm{d} s\right),
$$

where $f(t, T), 0 \leq t \leq T<\infty$, are instantaneous forward rates modeled by the stochastic differential equation $[\mathrm{SDE}]$

$$
\mathrm{d} f(t, T)=\alpha(t, T) \mathrm{d} t+\sigma(t, T) \mathrm{d} B_{t}, \quad 0 \leq t \leq T<\infty
$$


Here we require that $\sigma(\cdot, T)$ be a deterministic Borel-measurable function and $\alpha(\cdot, T)$ a predictable process for all $T$ wrt the $P$-completed filtration $\mathcal{F}_{t}$ generated by a one-dimensional Brownian motion $B_{t}, t \geq 0$.

Now, let us reparametrize the forward rates by the time-to-maturity $x=T-t$; that is, let us consider the forward curves

$$
f_{t}(x):=f(t, t+x)
$$

An application of the generalized Itô formula shows that under certain conditions on $\alpha(\cdot, T)$, $\sigma(\cdot, T)$ the forward curves $f_{t}(x)$ satisfy the first order SPDE

$$
\mathrm{d} f_{t}(x)=\frac{\mathrm{d}}{\mathrm{d} x} f_{t}(x)+\alpha_{t}(x) \mathrm{d} t+\sigma_{t}(x) \mathrm{d} B_{t},
$$

as in (Kunita 1997, Theorem 3.3.1). Here we use the notation $\alpha_{t}(x):=\alpha(t, t+x), \sigma_{t}(x):=$ $\sigma(t, t+x)$. Note that Equation (2.4) is referred to as the Musiela equation in the literature. See, e.g., (Carmona and Tehranchi 2006). See also (Da Prato and Zabczyk 1992) and the references therein for more information about SPDE's.

A deficiency of the model of Equation (2.4) is that it does not capture the feature of maturity-specific risk. A model with such a property would enable hedging of bond options with unique portfolio strategies. On the other hand, it would meet the intuitive requirement that maturities of the bonds underlying the bond option are used in the hedging portfolio.

A more realistic model than that of Equation (2.4), which takes into account maturityspecific risk, would consequently have the form

$$
\mathrm{d} f_{t}(x)=\frac{\mathrm{d}}{\mathrm{d} x} f_{t}(x)+\alpha_{t}(x) \mathrm{d} t+\sigma_{t}(x) \mathrm{d} B_{t}(x),
$$

where each noise $B_{t}(x)$ stands for the risk arising from the the time-to-maturity $x$. Here we may think of $B_{t}(x)$ as a Brownian sheet in $t$ and $x$. So Equation (2.5) can be recast as

$$
\mathrm{d} f_{t}(x)=\frac{\mathrm{d}}{\mathrm{d} x} f_{t}(x)+\alpha_{t}(x) \mathrm{d} t+\sum_{k \geq 1} \sigma_{t}^{(k)}(x) \mathrm{d} B_{t}^{(k)},
$$

where $\sigma^{(k)}(\cdot), k \geq 1$, are deterministic measurable functions and $B_{t}^{(k)}, k \geq 1$, are independent one-dimensional Brownian motions.

In what follows we want to assume that the forward curves are modeled by functions of a Hilbert space $H$. This space should exhibit the natural feature that evaluation functionals on it are continuous; that is,

$$
\begin{aligned}
\delta_{x}: H & \longmapsto \mathbb{R} \\
f & \longmapsto f(x)
\end{aligned}
$$

is continuous on $H$ for all $x$. Further, it is desirable that the generator $A:=\frac{\mathrm{d}}{\mathrm{d} x}$ in Equation (2.6) admits a strongly continuous semigroup $S_{t}$ on $H$. The semigroup $S_{t}$ is the left shift operator given by

$$
\left(S_{t} f\right)(x)=f(t+x)
$$

The following family $\left\{H_{w}\right\}$ of Hilbert spaces of Sobolev type introduced by (Filipović 2001) fulfills the above-mentioned conditions: Let $w:[0, \infty) \rightarrow(0, \infty)$ be a non-decreasing function such that

$$
\int_{0}^{\infty} \frac{1}{w(x)} \mathrm{d} x<\infty
$$


Then $H_{w}$ is defined as

$$
H_{w}=\left\{f:[0, \infty) \longrightarrow \mathbb{R} \mid f \text { is absolutely continuous and } \int_{0}^{\infty}\left(f^{\prime}(x)\right)^{2} w(x) \mathrm{d} x<\infty\right\},
$$

and is equipped with the scalar product

$$
\langle f, g\rangle_{H_{w}}=f(0) g(0)+\int_{0}^{\infty} f^{\prime}(x) g^{\prime}(x) w(x) \mathrm{d} x
$$

In the sequel we require that

$$
\alpha_{t}(\cdot), \sigma_{t}^{(k)}(\cdot) \in H \text {, a.e., } \quad \forall t \geq 0
$$

Consider the special case that $\alpha_{t}(x)=\alpha_{t}^{*}(x) f_{t}(x)$ for a deterministic function $\alpha_{t}^{*}(x)$. Then, using integrating factors we observe that the mild solution of the SDE of Equation (2.6) is explicitly given by the Gaussian random field

$$
\begin{aligned}
f_{t}(x)= & \exp \left(\int_{0}^{t} \alpha^{*}(s, t+x) \mathrm{d} s\right) f(0, t+x) \\
& +\sum_{k \geq 1} \int_{0}^{t} \exp \left(\int_{s}^{t} \alpha^{*}(u, t+x) \mathrm{d} u\right) \sigma^{(k)}(s, t+x) \mathrm{d} B_{t}^{(k)}
\end{aligned}
$$

Now, let $W_{t}$ be a $Q$-Wiener process, where $Q$ is a symmetric non-negative operator on a separable Hilbert space $U$ with $\operatorname{Trace}(Q)<\infty$. Set $U_{0}=Q^{1 / 2}(U)$, which is a Hilbert space with norm

$$
\|h\|_{0}:=\left\|Q^{-1 / 2}(h)\right\|, \quad u \in U_{0}
$$

Denote by $L_{2}(U, H)$ the space of Hilbert-Schmidt operators from $U$ to $H$ with the operator norm $\|\cdot\|_{L_{2}}$. Further, let $u_{k}, k \geq 1$, be an orthonormal basis of $U$, and suppose that there exists a Borel-measurable map

$$
\sigma:[0, T] \longrightarrow L\left(U_{0}, H\right)
$$

such that

$$
\sigma_{t}\left[Q^{1 / 2}\left(u_{k}\right)\right]=\sigma_{t}^{(k)}(\cdot)
$$

and

$$
\sigma_{t} \circ Q^{1 / 2} \in L_{2}(U, H)
$$

for all $(t, k)$ in Equation (2.6), where $\circ$ represents the composition of operators. Then we can view $\left\{B_{t}^{(k)}\right\}_{0 \leq t<T}, k \geq 1$, in Equation (2.6) as a Wiener process $B_{t}$ cylindrically defined on $U$, and rewrite Equation (2.6) as

$$
\mathrm{d} f_{t}=A f_{t}+\alpha_{t} \mathrm{~d} t+\sigma_{t} \mathrm{~d} W_{t}
$$

In the sequel we assume that there exists a predictable unique strong solution

$$
\left(t \longmapsto f_{t}(\cdot)\right) \in C([0, T] ; H)
$$

to Equation (2.10). 
Remark 2.1. Suppose that $\alpha_{t}=b\left(t, f_{t}\right)$ in Equation (2.10), where $b:[0, T] \times H \rightarrow H$ is a Borel-measurable map. Then the following set of conditions provide sufficient criteria for the existence of a unique strong solution of Equation (2.10).

(i) $f_{t}$ is a unique mild solution of Equation (2.10).

(ii) $f_{0} \in \operatorname{Dom}(A) ; S_{t-s} b(s, x) \in \operatorname{Dom}(A) ; S_{t-s} \sigma_{s} u \in \operatorname{Dom}(A), \forall u \in U_{0}, t \geq s$.

(iv)

$$
\left\|A S_{t-s} b(s, x)\right\|_{H} \leq q(t-s)\|x\|_{H} \text {, for some } q \in L^{1}\left([0, T] ; \mathbb{R}_{+}\right) .
$$

See, e.g., (Kai 2006).

Assume that $\sigma_{t}$ is invertible for all $0 \leq t \leq T$ a.e. and that the integrability condition

$$
\sup _{t \in[0, T]} \mathrm{E}\left[\exp \left(\delta\left\|\sigma_{t}^{-1}\left[A f_{t}+\alpha_{t}\right]\right\|_{0}^{2}\right)\right]<\infty
$$

holds for some $\delta>0$. Then Girsanov's Theorem [see, e.g., (Bensoussan 1971)] applied to Equation (2.10) entails that

$$
\mathrm{d} f_{t}=\sigma_{t} \mathrm{~d} \widehat{W}_{t}
$$

where

$$
\widehat{W}_{t}=W_{t}-\int_{0}^{t} \psi(s) \mathrm{d} s
$$

is a $Q$-Wiener process under the change of measure $\widehat{P}$ given by

$$
\widehat{P}(A)=\mathrm{E}\left[\mathbf{1}_{A} \exp \left(\int_{0}^{T}\left\langle\psi(s), \mathrm{d} W_{s}\right\rangle_{0}-\frac{1}{2} \int_{0}^{T}\|\psi(s)\|_{0}^{2} \mathrm{~d} s\right)\right],
$$

with

$$
\psi(t):=\sigma_{t}^{-1}\left[A f_{t}+\alpha_{t}\right]
$$

Consequently $f_{t}$ is a Gaussian $\mathcal{F}_{t}$-martingale with respect to $\widehat{P}$. Define

$$
\widehat{f}_{t}=f_{t}-f_{0}=\int_{0}^{t} \sigma_{s} \mathrm{~d} \widehat{W}_{s}
$$

Thus $\widehat{f}_{t}(x)$ is a centered Gaussian random field wrt time and time-to-maturity under $\widehat{P}$. We wish to use these forward curves to define an expanded concept of duration which serves as a tool to measure interest rate sensitivities of bond options or bond portfolios with respect to the whole yield surface

$$
(t, x) \longmapsto f_{t}(x)
$$

In view of the relation between Malliavin derivatives and Gateaux derivatives it is reasonable to define the duration of an interest rate instrument as the Malliavin derivative of a square integrable functional of $\widehat{f}_{t}(x)$. To this end we have to introduce a Malliavin calculus with 
respect to $\widehat{f}_{t}(x)$, which is the centered forward curve in the risk neutral world. For this purpose let $(\Omega, \widehat{\mathcal{F}}, \widehat{P})$ be our reference probability space, where $\widehat{\mathcal{F}}$ is generated by $\widehat{f}_{t}(x)$. In the following, denote by $I$ the index set with respect to the -tuples $(t, x)$, and set $\widehat{f}(u)=\widehat{f}_{t}(x)$ if $u=(t, x) \in I$. Let

$$
C(u, r)=\mathrm{E}[\widehat{f}(u) \widehat{f}(r)]
$$

be the covariance function of $\widehat{f}$. Further consider the reproducing kernel Hilbert space [RKHS] $K$ of $C$ with norm $\|\cdot\|_{K}$. See, e.g., (Chatterji and Mandrekar 1978). Then $K$ is isometrically isomorphic to the closure of the linear span of $\widehat{f}(u), u \in I \in L^{2}(\Omega, \widehat{\mathcal{F}}, \widehat{P})$. Using in addition the continuity of evaluation functionals on $H$ and the theorem of Banach-Steinhaus we find that $K$ is isometrically isomorphic to the space

$$
H(\widehat{f}):=\left\{\lambda:[0, T] \longrightarrow H^{*} \text { Borel measurable } \mid \int_{0}^{T}\left\|\lambda_{s} \circ \sigma_{s}\right\|_{L_{2}^{0}}^{2} \mathrm{~d} s<\infty\right\},
$$

where $\|B\|_{L_{2}^{0}}:=\left\|B \circ Q^{1 / 2}\right\|_{L^{2}}<\infty$ for $B \in L(H, H)$. Here $H^{*}$ stands for the topological dual of $H$.

By (Chatterji and Mandrekar 1978) we obtain the following chaos decomposition.

$$
L^{2}(\Omega, \widehat{\mathcal{F}}, \widehat{P})=\sum_{p \geq 0} \oplus I_{p}\left(K^{\widehat{\otimes} p}\right),
$$

where $K^{\widehat{\otimes} p}$ is the $p$-fold symmetric tensor product of $K$, and where $I_{p}: K^{\otimes p} \rightarrow L^{2}(\Omega, \widehat{\mathcal{F}}, \widehat{P})$ are linear operators such that the the following properties hold.

$$
\begin{gathered}
\mathrm{E}\left[I_{p}(f)\right]=0 \\
\mathrm{E}\left[I_{p}(f) I_{q}(g)\right]= \begin{cases}0 & , p \neq q \\
p !\langle\widetilde{f}, \widetilde{g}\rangle_{K} & , p=q\end{cases}
\end{gathered}
$$

for $f \in K^{\otimes p}, g \in K^{\otimes q}$, where $\tilde{f}$ is the symmetrization of $f$. Here $I_{p}$ is recursively defined by

$$
I_{p+1}(g h)=I_{p}(g) I_{1}(h)-\sum_{k=1}^{p} I_{p-1}(g \underset{k}{\otimes} h)
$$

for $g \in K^{\otimes p}, h \in K$, where

$$
I_{1}(h):=\int_{0}^{T} h_{s} \mathrm{~d}\left(\sigma_{s} \widehat{W}_{s}\right)=\int_{0}^{T} h_{s} \circ \sigma_{s} \mathrm{~d} \widehat{W}_{s} .
$$

for $h \in H(\widehat{f})$. See (Mandrekar and Zhang 1993).

Now let $u \in L^{2}(\Omega ; K)$ and let $u_{t}$ have the chaos representation

$$
u_{t}=\sum_{p \geq 0} I_{p}\left(f_{p}^{t}\right)
$$

for unique $f_{p}^{t} \in K^{\widehat{\otimes} p}$ and each $t \in I$. Denote by $\widetilde{f}_{p}$ the symmetrization of an appropriate version of $f_{p}^{t}\left(t_{1}, \ldots, t_{p}\right)$ wrt $t_{1}, \ldots, t_{p}$, and $t$. Then the Skorohod integral of the process $u_{t}$ is 
defined as

$$
\delta(u .)=\sum_{p \geq 1} I_{p+1}\left(\widetilde{f}_{p}\right)
$$

if

$$
\sum_{p \geq 1}(p+1) !\left\|\widetilde{f}_{p}\right\|_{K \widehat{\otimes} p+1}^{2}<\infty
$$

is fulfilled.

The Malliavin derivative $D_{u} F \in L^{2}(\Omega ; K)$ of a square integrable functional $F$ of the forward curve $\widehat{f}$ can be defined as the adjoint operator of $\delta$ in Equation (2.16). In the sequel we shall denote by $\mathbb{D}^{1,2} \subset L^{2}(\Omega, \widehat{\mathcal{F}}, \widehat{P})$ the domain of the Malliavin derivative $D$.

In view of the financial applications we have in mind it is important to note that the Malliavin derivative can be regarded as a sensitivity measure with respect to the fluctuations of the yield surface $(t, x) \longmapsto f_{t}(x)$. The latter can be justified by the following relationship between the Malliavin derivative and the stochastic Gateaux $K$-derivative: Let $X$ be the support of the image measure $\mu$ of $\widehat{f}$ under $\widehat{P}$ in $C([0, T] ; H)$. Then by (Borel 1976) we find that $X$ is the closure of $K$ in $C([0, T] ; H)$. Further, in (Gawarecki and Mandrekar 1993, Proposition 4.1) we have that if for $F \in L^{2}(\mu)$

$$
\frac{F(x+\epsilon k)-F(x)}{\epsilon}
$$

converges in $L^{2}(\mu)$ as $\epsilon \rightarrow 0$ for $k \in K$, then $D . F \in L^{2}(\mu ; K)$ exists and the above limit equals $(D . F, k)_{K}$.

Since the measure $P$ in Equation (2.3) is equivalent to $\widehat{P}$ we see that the convergence of Expression (2.17) to $\langle D . F, k\rangle_{K}$ also holds in probability with respect to the image measure of the forward curves under the original measure $P$. Therefore, if $F=\xi_{T}$ is the terminal value of a bond portfolio, we may interpret the Malliavin derivative D.F as a sensitivity measure of the fluctuations of the whole yield surface in this portfolio. The latter observation gives rise to introduce an expanded concept of duration as follows.

Definition 2.1 (Stochastic duration). Let $F$ be a square integrable functional of the forward curve $\widehat{f}$ wrt $\widehat{P}$. Assume that $F$ is Malliavin differentiable wrt $\widehat{f}$. Then the stochastic duration of $F$ is stochastic process

$$
D . F \in L^{2}(\Omega, \widehat{\mathcal{F}}, \widehat{P} ; K)
$$

Remark 2.2. We shall mention that we also could have introduced our concept of stochastic duration wrt mild solutions $f_{t}$ of Equation (2.10). In this case one can replace Condition (2.11) by assuming that

$$
\sup _{t \in[0, T]} \mathrm{E}\left[\exp \left(\delta\left\|\sigma_{t}^{-1}\left[\alpha_{t}\right]\right\|_{0}^{2}\right)\right]<\infty
$$

for some $\delta>0$. Compared to mild solutions, strong solutions are rare. However, from the viewpoint of applications we have in mind it is technically more convenient to deal with strong solutions. See Section 3.

We want to illustrate this concept by calculating the stochastic duration of certain interest rate claims. For this purpose we need the following auxiliary results.

The first Lemma gives a chain rule for the Malliavin derivative $D$. 
Lemma 2.2 (Chain Rule). Let $F$ be Malliavin differentiable with respect $\widehat{f}$, i.e., $F \in \mathbb{D}^{1,2}$. Further, suppose that $g: \mathbb{R} \rightarrow \mathbb{R}$ is continuously differentiable with bounded derivative. Then $g(F) \in \mathbb{D}^{1,2}$ and

$$
D_{u} g(F)=g^{\prime}(F) D_{u} F
$$

for each $u \in K$. Here $g^{\prime}$ stands for the derivative of $g$.

Proof. The proof follows from arguments in the Brownian motion case. See (Di Nunno, Øksendal, and Proske 2008, Theorem 3.5) or (Nualart 1995, Proposition 1.2.2).

The next Lemma pertains to the closability of the Malliavin derivative.

Lemma 2.3 (Closability). Let $F \in L^{2}(\widehat{P})$ and $\left(F_{k}\right)_{k \geq 1} \subset \mathbb{D}^{1,2}$ such that

$$
F_{k} \underset{k \rightarrow \infty}{\longrightarrow} F \text { in } L^{2}(\widehat{P})
$$

and

$$
\text { D. } F_{k} \text { converges in } L^{2}(\widehat{P} ; K)
$$

Then $F \in \mathbb{D}^{1,2}$ and

$$
D . F_{k} \underset{k \rightarrow \infty}{\longrightarrow} D . F \text { in } L^{2}(\widehat{P} ; K)
$$

Proof. See the arguments in (Di Nunno, Øksendal, and Proske 2008, Theorem 3.3).

Example 2.1 (Zero Coupon Bond). As before let $P(t, T)$ be the price at time $t$ of a zero coupon bond, which pays $\$ 1$ at maturity $T$. Then using the instantaneous forward rates $f(t, s)$, $0 \leq t \leq s$, we have that

$$
\begin{aligned}
P(t, T) & =\exp \left(-\int_{t}^{T} f(t, s) \mathrm{d} s\right) \\
& =\exp \left(-\int_{0}^{T-t} f_{t}(x) \mathrm{d} x\right)
\end{aligned}
$$

We find that

$$
\begin{aligned}
D_{r, y}\left(\int_{0}^{T-t} f_{t}(x) \mathrm{d} x\right) & =\int_{0}^{T-t} D_{r, y}\left(f_{t}(x)\right) \mathrm{d} x \\
& =\int_{0}^{T-t} \mathbf{1}_{[0, t]}(r) \mathrm{d} x \\
& =(T-t) \mathbf{1}_{[0, t]}(r),
\end{aligned}
$$

where $\mathbf{1}_{[0, t]}$ is the indicator function of $[0, t]$. Then the chain rule of Lemma 2.2 (in connection with Lemma 2.3) shows that the stochastic duration D.P $(t, T)$ of $P(t, T)$ in the HJM model is given by

$$
D_{r, y} P(t, T)= \begin{cases}-(T-t) P(t, T) & , \text { if } 0 \leq r \leq t \\ 0 & , \text { otherwise }\end{cases}
$$


So $D_{r, y} P(t, T) / P(t, T), 0 \leq r \leq t$, has the form of the classical duration in Section 1. The latter expression seems to suggest that we should rather use D.F/F as a generalized duration than D.F. However, a general interest rate claim $F$ may be zero for a positive probability. Therefore it is reasonable to introduce D.F as an expanded concept of duration. Note that our definition does not generalize Macaulay's duration in the sense that D.F gives the classical duration if the interest rate claim $F$ is deterministic, that is, a functional of a deterministic (piecewise flat) yield surface. The explanation for this is that the duration concepts are based on different interest rate models.

The classical duration presumes yield surfaces which are flat or piecewise flat. Such a model is fundamentally different from a stochastic interest rate model. For example, under our conditions yield surfaces in our [risk-neutral] HJM model only assume a certain constant value with probability zero. In view of this we may therefore consider the stochastic duration as a concept which is analogous to the classical one in the HJM setting.

Example 2.2 (Interest Rate Cap). Consider a cap of the form

$$
F=(R(t, T)-K)^{+},
$$

where $K$ is the cap rate and $R(t, T)$ the average interest rate given by

$$
R(t, T)=\frac{1}{T-t} \int_{t}^{T} r(s) \mathrm{d} s
$$

Here $r(t)=f(t, t)$ is the overnight interest rate, also known as the short rate. We observe that

$$
\begin{aligned}
D_{r, y}\left(\frac{1}{T-t} \int_{t}^{T} r(s) \mathrm{d} s\right) & =\frac{1}{T-t} \int_{t}^{T} D_{r, y}(r(s)) \mathrm{d} s \\
& =\frac{1}{T-t} \int_{t}^{T} D_{r, y}\left(f_{s}(0)\right) \mathrm{d} s \\
& =\mathbf{1}_{[0, t]}(r)
\end{aligned}
$$

Now let us approximate the $\varphi(x):=(x-K)^{+}$by functions $\left\{\varphi_{n}\right\}$ with

$$
\varphi_{n}(x)=\varphi(x) \text { for }|x-K| \geq \frac{1}{n}
$$

and

$$
0 \leq \varphi_{n}^{\prime}(x) \leq 1 \text { for all } x
$$

Then it follows from Lemma 2.2 and Lemma 2.3 that

$$
D_{r, y} F=\mathbf{1}_{[K, \infty)}(R(t, T)) \cdot \mathbf{1}_{[0, t]}(r)
$$


Example 2.3 (Asian Option). Let us also have a look at the following Asian type of option defined as

$$
F=\frac{1}{\left(\bar{x}_{2}-\bar{x}_{1}\right)\left(T_{2}-T_{1}\right)} \int_{\bar{x}_{1}}^{\bar{x}_{2}} \int_{T_{1}}^{T_{2}} f_{t}(x) \mathrm{d} t \mathrm{~d} x
$$

Then

$$
\begin{aligned}
D_{r, y} F & =\frac{1}{\left(\bar{x}_{2}-\bar{x}_{1}\right)\left(T_{2}-T_{1}\right)} \int_{\bar{x}_{1}}^{\bar{x}_{2}} \int_{T_{1}}^{T_{2}} \mathbf{1}_{[0, t]}(r) \mathrm{d} t \mathrm{~d} x \\
& =\mathbf{1}_{[0, t]}(r)
\end{aligned}
$$

3. Estimation of Stochastic Duration

and the Construction of Immunization Strategies

In the previous section we introduced the concept of stochastic duration $D_{t, y} F$ and gave examples of interest rate derivatives $F$ whose stochastic duration can be computed explicitly. In general, the stochastic duration of an interest claim or a complex bond portfolio cannot be determined explicitly. The latter is also due to the fact that, e.g., a dynamically hedged bond portfolio is a stochastically weighted sum of interest rate claims. The weights of the portfolio or hedging strategy at any time point are usually complicated functionals of the stochastic forward curve. In order to overcome this deficiency we aim at resorting to an estimate of $D_{t, y} F$. A reasonable estimate of $D_{t, y} F$ could be the expected stochastic duration of $F$ given the observed forward curves $\widehat{f}_{s}, 0 \leq s \leq t$. This estimate naturally appears in the Clark-Ocone formula or as a solution of a backward stochastic differential equation [BSDE].

Using the fact that the set

$$
\left\{\exp \left\{I_{1}(h)-\frac{1}{2}\|h\|_{K}^{2}\right\} \mid h \in K\right\}
$$

is total in $L^{2}(\Omega, \widehat{\mathcal{F}}, \widehat{P})$ one finds in connection with Relation (2.15) the Clark-Ocone formula wrt the forward curves $\widehat{f}_{t}$ takes the following form. See also(Di Nunno, Øksendal, and Proske 2008).

$$
F=\mathrm{E}_{\widehat{P}}[F]+\int_{0}^{T} \mathrm{E}\left[D_{s}^{*}(F) \mid \widehat{\mathcal{F}}_{s}\right] \mathrm{d} \widehat{f}_{s},
$$

where the $\mathcal{B}([0, T]) \otimes \widehat{\mathcal{F}}, \mathcal{B}\left(H^{*}\right)$-measurable map $D_{.}^{*}(F):[0, T] \times \Omega \rightarrow H^{*}$ can be linearly isometrically identified with the Malliavin derivative, i.e., stochastic duration, D.F in Definition 2.1. Further, $F \in L^{2}(\Omega, \widehat{\mathcal{F}}, \widehat{P})$ is in the domain of $D^{*}$ and $\widehat{\mathcal{F}}_{t}$ is the $\widehat{P}$-completed filtration generated by $\widehat{f}_{s}, 0 \leq s \leq t$.

The $H^{*}$-valued conditional expectation

$$
\mathrm{E}\left[D_{t}^{*}(F) \mid \widehat{\mathcal{F}}_{t}\right], \quad 0 \leq t \leq T
$$


can be regarded as an estimation of D.F. Now let us us have a look at the BSDE

$$
Y_{t}=Y_{T}-\int_{t}^{T} Z_{s} \mathrm{~d} \widehat{f_{s}},
$$

where $Y_{T}=F$. Then we observe that

$$
Z_{t}=\mathrm{E}\left[D_{t}^{*}(F) \mid \widehat{\mathcal{F}}_{t}\right] \quad \widehat{P} \text { a.e. }
$$

for $0 \leq t \leq T$, a.e.

We wish to recast the dynamics of the solution $\left(Y_{t}, Z_{t}\right)$ in Equation (3.1) wrt the original measure $P$. Since $\sigma_{t}$ is invertible $t$-a.e. we see that the natural filtration of $\widehat{W}_{t}$ coincides with the filtration $\widehat{\mathcal{F}}_{t}$. Assume that there exists a unique strong solution $f_{t}^{*}$ of the SPDE

$$
f_{t}^{*}=\int_{0}^{t} \sigma_{s}^{-1}\left[A f_{s}^{*}+\alpha_{s}(s, \cdot)\right] \mathrm{d} s+W_{t}, \quad 0 \leq t \leq T,
$$

where $W_{t}$ is the $Q$-cylindrical Wiener process in Equation (2.12). See, e.g., (Prévôt and Röckner 2007) for criteria about the existence and uniqueness of solutions of non-linear SPDE's.

Remark 3.1. Let $\alpha_{t}=b\left(t, f_{t}\right)$ in Equation (3.2) for a Borel measurable map $b:[0, T] \times H \rightarrow$ $H$. Impose on $A$ the rather strong condition to be a bounded operator on $H$. Further assume that the drift coefficient $F(t, x):=\sigma_{t}^{-1}[A x+b(t, x)]$ satisfies a linear growth and Lipschitz condition wrt $x$, uniformly in $t$. Then the Picard iteration gives a unique strong solution of Equation (3.2).

The Assumption of Equation (3.2) entails that the natural filtration of $W_{t}$ is given by $\widehat{\mathcal{F}}_{t}$. Then it follows from Equation (2.12) that the solution $\left(Y_{t}, Z_{t}\right)$ in Equation (3.1) has the following BSDE dynamics under $P$.

$$
\begin{aligned}
Y_{t} & =Y_{T}+\int_{t}^{T} Z_{s}\left[A f_{s}+\alpha_{s}(s, \cdot)\right] \mathrm{d} s-\int_{t}^{T} Z_{s} \mathrm{~d} W_{s}^{*} \\
Y_{T} & =F,
\end{aligned}
$$

where $W^{*}$ is the square integrable $H$-valued martingale given by

$$
W_{t}^{*}=\int_{0}^{t} \sigma_{s} \mathrm{~d} W_{s}
$$

So we see that the estimate $Z_{t}$ of the stochastic duration of $F$ satisfies the forward-backward stochastic partial differential equation [FBSPDE]

$$
\begin{aligned}
\mathrm{d} f_{t} & =A f_{t}+\alpha_{t} \mathrm{~d} t+\sigma_{t} \mathrm{~d} W_{t} \\
Y_{t} & =Y_{T}+\int_{t}^{T} Z_{s}\left[A f_{s}+\alpha_{s}(s, \cdot)\right] \mathrm{d} s-\int_{t}^{T} Z_{s} \mathrm{~d} W_{s}^{*} \\
Y_{T} & =F,
\end{aligned}
$$


where $F$ is a measurable functional of the solution of the forward SPDE, i.e., of the forward curves $f_{t}$. For more information about linear forward-backward S(P)DE's the reader may consult (Ma and Yong 1999). See also (Øksendal, Proske, and Zhang 2005).

Remark 3.2. In view of financial applications it would be desirable to develop a numerical approximation scheme for solutions $\left(Y_{t}, Z_{t}\right)$ of FBSPDE's of the type of Equation (3.3). In general, this is a challenging task. A possible ansatz to this problem (in some special cases) would be to employ the results in (Zhang 2004) or in (Nakayama 2002) in connection with Galerkin approximation. Another approach could be based on finite element or finite difference schemes in a backward stochastic partial differential equation [BSPDE] setting. In the framework of the linear Gaussian model, as in Equation (2.9), for the forward curves one can simplify further the numerical analysis by using dimension reduction techniques as, e.g., principal component analysis of interest rate data. See (Carmona and Tehranchi 2006).

Remark 3.3. Using stochastic distribution theory the concept of stochastic duration for interest rate claims $F \in \mathbb{D}^{1,2}$ can be extended to the case of claims contained in a space of generalized random variables which comprises the space of square integrable functionals of the forward curves wrt $\widehat{P}$. See, e.g., (Üstünel 1995) or (Da Prato and Zabczyk 1992). As a consequence we may still interpret $Z_{t}$ in Equation (3.3) as an estimate of the stochastic duration of a claim $F$, when $F \in L^{2}(P) \cap L^{2}(\widehat{P})$.

Finally, we want to discuss an extension of the concept of delta hedge of interest rate sensitive securities developed by (Hull and White 1994) to a stochastic setting, which involves the fluctuations of the whole yield surface. The purpose of delta hedge is to immunize portfolios of interest-rate-sensitive securities under Ho's interest rate scenario (Ho 1992). In other words, the idea devised by (Hull and White 1994) is to neutralize given financial positions in interestrate derivatives against parallel shifts of $i$-years spot rates (or key rates).

We want to propose a mathematical framework which facilitates the construction of immunization strategies of interest-rate-sensitive portfolios in the sense of (Hull and White 1994) wrt stochastic fluctuations of the yield surface. In fact, we aim at minimizing the exposure of given financial positions to interest rate risk by going short in bonds of a generalized bond portfolio, that is, of self-financing portfolios composed of infinitely many bonds of any maturity.

To this end we need some notions and conditions. Suppose that the generalized HJM-model [see Equation (2.10)] for the forward curves $f_{t}$ fulfills the HJM no-arbitrage condition

$$
\alpha_{t}(x)=\sum_{k \geq 1} \sigma_{t}^{(k)}(x)\left(I_{x}\left(\sigma_{t}^{(k)}\right) \int_{0}^{x} \sigma_{t}^{(k)}(u) \mathrm{d} u+\lambda_{t}^{(k)}\right),
$$

where the processes $\lambda_{t}^{(k)}, k \geq 1$, are the Fourier coefficients of a predictable $H$-valued process

$$
\lambda_{t}=\sum_{k \geq 1} \lambda_{t}^{(k)} e_{k}
$$

Here $\left\{e_{k}\right\}$ is an optimal normal basis of $H$. Further $\sigma_{t}^{(k)}, k \geq 1$, is given as in Equation (2.6) and $I_{x}$ is a linear functional in $H^{*}$ defined by

$$
I_{x}(f)=\int_{0}^{x} f(u) \mathrm{d} u
$$


We remark that the processes $\lambda_{t}^{(k)}, k \geq 1$, admit the interpretation of market prices of risk wrt different bond maturities.

Now let us consider the discounted bond price curve $\widetilde{P}_{t}(\cdot)$ given by

$$
\widetilde{P}_{t}(x)=\exp \left(-\int_{0}^{t} f_{s}(0) \mathrm{d} s-\int_{0}^{x} f_{s}(x) \mathrm{d} s\right)
$$

We require that the conditions

$$
\mathrm{E}\left[\exp \left(\int_{0}^{t}\left\langle\lambda_{s}, \mathrm{~d} W_{s}\right\rangle_{0}-\frac{1}{2} \int_{0}^{t}\left\|\lambda_{s}\right\|_{0}^{2} \mathrm{~d} s\right)\right]=1
$$

and

$$
\int_{0}^{t}\left(\int_{0}^{s}\left\|\delta_{s-u} \circ \sigma_{s}\right\|_{L_{2}^{0}}^{2} \mathrm{~d} u\right)^{1 / 2} \mathrm{~d} s<\infty
$$

hold for all $t \geq 0$.

Then, using Itô's Formula and Girsanov's Theorem one finds that

$$
\widetilde{P}(t, T)=P(0, T)-\int_{0}^{t} P(s, T) I_{T-s} \circ \sigma_{s} \mathrm{~d} \widetilde{W}_{s},
$$

where

$$
\widetilde{W}_{t}=W_{t}+\int_{0}^{t} \lambda_{s} \mathrm{~d} s
$$

is a $Q$-Wiener process under a local martingale measure $\widetilde{P}$.

Define

$$
\tilde{\sigma}_{t}(\omega, x)=P_{t}(x) I_{x} \circ \sigma_{t}
$$

Let $G$ be a separable Hilbert space in $C([0, \infty))$ such that evaluation functionals $\delta_{x}$ on $G$ are continuous and the semigroup $S_{t}$ of left shift operators is strongly continuous on $G$. See Equations (2.7) and (2.8). From now forward we assume that $\widetilde{\sigma}_{t}$ in Equation (3.5) is a predictable $L\left(U_{0}, G\right)$-valued process such that $\int_{0}^{T}\left\|\widetilde{\sigma}_{s}\right\|_{L_{2}^{0}}^{2} \mathrm{~d} s<\infty$ a.e. The latter implies that the bond price curves $\widetilde{P}_{t}$ are $G$-valued and satisfy

$$
\mathrm{d} \widetilde{P}_{t}=A \widetilde{P}_{t} \mathrm{~d} t-\widetilde{\sigma}_{t} \mathrm{~d} \widetilde{W}_{t}
$$

or

$$
\mathrm{d} \widetilde{P}_{t}=\left(A \widetilde{P}_{t}-\widetilde{\sigma}_{t}\left[\lambda_{t}\right]\right) \mathrm{d} t-\widetilde{\sigma}_{t} \mathrm{~d} W_{t}
$$

in the mild sense.

Now let us consider generalized bond portfolios. See (Björk, Masi, Kabanov, and Runggaldier 1997). That is, the wealth process $V_{t}$ of such portfolios is given by

$$
V_{t}=V_{t}(\phi):=\phi_{t}\left[P_{t}(\cdot)\right], \quad t \geq 0,
$$


where $\phi_{t}$ is a predictable $G^{*}$-valued process. The process $\phi_{t}$ can be regarded as the trading strategy of an investor who manages a portfolio with infinitely many bonds of any maturity. For example, the strategy $\phi_{t}=\delta_{T-t}$ stands for buying and holding a zero-coupon bond with maturity $T$, since $\phi_{t}\left[P_{t}(\cdot)\right]=P(0, T)$.

Assume that

$$
\mathrm{E}_{\widetilde{P}}\left[\int_{0}^{t}\left\|\phi_{s} \circ \widetilde{\sigma}_{s}\right\|_{L_{2}^{0}}^{2} \mathrm{~d} s\right]<\infty
$$

for all $t \geq 0$. Then we shall say that a trading strategy $\phi_{t}, t \geq 0$, is self-financing if there is a $V_{0} \in \mathbb{R}$ such that

$$
\widetilde{V}_{t}(\phi)-\int_{0}^{t} \phi_{s} \circ \widetilde{\sigma}_{s} \mathrm{~d} \widetilde{W}_{s}=V_{0}
$$

for all $t \geq 0$ a.e. where $\widetilde{V}_{t}(\phi)$ is the discounted wealth process given by

$$
\widetilde{V}_{t}(\phi)=\phi_{t}\left[\widetilde{P}_{t}(\cdot)\right]
$$

See, e.g., (Björk, Masi, Kabanov, and Runggaldier 1997). We denote the set of all self-financing strategies by $\mathcal{A}$.

Remark 3.4. In the infinite-dimensional HJM-framework the existence of a unique martingale measure does not imply in general that the bond market given by Equation (3.4) is complete. The latter is a deficiency not shared by finite-rank models. However, since the kernels of $\widetilde{\sigma}_{t}$, as in Equation (3.5), are zero t-a.e. our bond market is approximately complete in the following sense. For all $\epsilon>0$ there exists a strategy $\phi^{\epsilon} \in \mathcal{A}$

$$
\mathrm{E}_{\widetilde{P}}\left[\left(\mathrm{E}_{\widetilde{P}}[\widetilde{h}]+\int_{0}^{T} \phi_{s}^{\epsilon} \circ \widetilde{\sigma}_{s} \mathrm{~d} \widetilde{W}_{s}-\widetilde{h}\right)^{2}\right]<\epsilon,
$$

where $\widetilde{h}$ a discounted contingent claim. See, e.g., (Björk, Masi, Kabanov, and Runggaldier 1997).

Suppose that a trader is long in interest rate securities at time $t \geq 0$ whose price process is $L_{t}$. In order to neutralize the risk coming from the fluctuations of the yield surface the trader wishes to go short in the generalized bond portfolio, as in Equation (3.6), for a self-financing strategy $\phi^{*} \in \mathcal{A}$ such that $\phi^{*}$ minimizes at any time point the worst-scenario interest rate sensitivity of the resulting portfolio. More precisely, the trader tries to find a $\phi^{*} \in \mathcal{A}$ such that

$$
\inf _{\phi^{*} \in \mathcal{A}} \mathrm{E}\left[\int_{0}^{T}\left\|D \cdot\left(L_{t}-V_{t}(\phi)\right)\right\|_{K}^{2} \mathrm{~d} t\right]=\mathrm{E}\left[\int_{0}^{T}\left\|D \cdot\left(L_{t}-V_{t}\left(\phi^{*}\right)\right)\right\|_{K}^{2} \mathrm{~d} t\right]<\infty,
$$

where $K$ is the RKHS of the forward curves. Note that

$$
\sup _{\|k\|_{K}=1}\langle D . F, k\rangle_{K}=\|D \cdot F\|_{K}
$$

for an interest claim $F \in \mathbb{D}^{1,2}$. So Equation (2.17) admits the interpretation that $\|D . F\|_{K}$ is the worst-scenario sensitivity with respect to all directional interest changes $k \in K$. 
Using the estimate $Z .=Z .(F)$ for the stochastic duration $D .(F)$ in the FBSPDE of Equation (3.3) for $F=L_{t}-V_{t}(\phi)$ [see Remark 3.3 and Relation 2.15] the optimization problem of Equation (3.7) then takes the form

$$
\begin{aligned}
& \inf _{\phi^{*} \in \mathcal{A}} \mathrm{E}\left[\int_{0}^{T} \int_{0}^{T}\left\|Z_{u}\left(L_{t}-V_{t}(\phi)\right) \circ \sigma_{u}\right\|_{L_{2}^{0}}^{2} \mathrm{~d} u \mathrm{~d} t\right] \\
& =\mathrm{E}\left[\int_{0}^{T} \int_{0}^{T}\left\|Z_{u}\left(L_{t}-V_{t}\left(\phi^{*}\right)\right) \circ \sigma_{u}\right\|_{L_{2}^{0}}^{2} \mathrm{~d} u \mathrm{~d} t\right]<\infty
\end{aligned}
$$

for $\phi^{*} \in \mathcal{A}$.

We see that the construction of an immunized bond portfolio reduces to an optimal control problem of the FBSPDE of Equation (3.3) or the FBSPDE

$$
\begin{aligned}
\widetilde{V}_{t}(\phi) & =\widetilde{V}_{0}(\phi)-\int_{0}^{t} \phi_{s} \circ \widetilde{\sigma}_{s} \mathrm{~d} \widetilde{W}_{s} \\
Y_{t} & =Y_{T}+\int_{t}^{T} Z_{s}\left[A f_{s}+\alpha_{s}(s, \cdot)\right] \mathrm{d} s-\int_{t}^{T} Z_{s} \mathrm{~d} W_{s}^{*} \\
Y_{T} & =F,
\end{aligned}
$$

where $F=L_{t}-V_{t}(\phi)$ for each $t$, if $L_{t}$ is a measurable functional of $\widetilde{V} .(\phi)$.

An approach to tackle this problem could be based on a stochastic maximum principle for FBSPDE's. See (Haadem and Mandrekar 2010). From a practical point of view it would be important to find numerical approximation schemes for a delta hedge $\phi^{*} \in \mathcal{A}$.

\section{Remark 3.5.}

(1) It is conceivable that the concept of g-expectation by (Peng 1997) for BSDE's can be generalized to FBSPDE's of the type of Equation (3.3). The latter would enable the construction of risk measures of functionals of forward curves. Such a construction would reveal the role of the stochastic duration as a building block for general interest rate risk measures.

(2) We point out that our framework also allows for the definition of stochastic convexity, that is, a measure of "curvature" wrt the fluctuations of the yield surface. It makes sense to define the stochastic duration of a twice Malliavin differentiable interest rate claim $F$ as

$$
D . D .(F) \in L^{2}(\Omega, \widehat{\mathcal{F}}, \widehat{P} ; K \otimes K)
$$

\section{ACKNOWLEDGMENT}

The authors wish to thank Vidyadhar S. Mandrekar for his valuable comments on this work. 


\section{Appendix A. Macaulay duration And portfolio immunization}

A.1. Macaulay duration. Consider the discrete and continuous cases separately.

A.1.1. Discrete case. In Macaulay's original concept duration was the weighted average by present value of the number of periods to maturity for a series of cash flows, typically those of interest and principal payments for a bond, normalized by the total present value (Macaulay 1938). For notation, let $V$ be the present value (or price) of the bond, $r>0$ be the [constant] rate of interest, and $n$ be the number of periods to maturity. The expression

$$
\mathcal{A}(r, n)=\frac{1-(1+r)^{-n}}{r}
$$

is the closed form for the present value of an annuity in arrears for $n$ periods at rate $r$, reflecting the typical payment scheme of a bond, e.g., a United States Treasury bond. Therefore the Macaulay duration $d_{\mathrm{Mac}}$ has the following definition for equally spaced cash flows of size $C$ and return of principal $P$.

$$
d_{\mathrm{Mac}}:=\frac{C \sum_{k=1}^{n} k(1+r)^{-k}+n P(1+r)^{-n}}{C \sum_{k=1}^{n}(1+r)^{-k}+P(1+r)^{-n}},
$$

or

$$
d_{\mathrm{Mac}}=-(1+r) \frac{\partial}{\partial r} \log \left[C \cdot \mathcal{A}(r, n)+P(1+r)^{-n}\right]
$$

In the simple case of a single cash flow - a zero coupon bond - Macaulay duration reduces to the number of periods $n$ to that payment, justifying the name.

Soon, however, practitioners began preferring a version of duration as the simple negative of the derivative of $V$ with respect to $r$, dropping the factor $(1+r)$. This version became known as the modified duration $d_{\text {mod }}$, with this definition.

$$
d_{\text {mod }}:=-\frac{\partial}{\partial r} \log \left[C \cdot \mathcal{A}(r, n)+P(1+r)^{-n}\right]
$$

Such redefinition provides the relationship

$$
d_{\mathrm{Mac}}=(1+r) d_{\mathrm{mod}},
$$

so that the modified duration of a zero coupon bond is $(1+r) n$.

In ordinary parlance, either form of duration is stated as a positive number, e.g., "The duration of this bond is ten years," as indicated. A rationale exists, however, for stating the duration as a negative number, reflecting the inverse relationship between changes in the level of interest and changes in price. Such versions, inverting the minus signs of Equations (A.1) and (A.2), more typically appear in Taylor series expansions of bond price, and in more developed mathematical expositions. The latter approach is assumed in this paper.

A.1.2. Continuous case. The continuous case is a straightforward extension of the discrete case. Let $C$, as previously, be the cash flow assigned to a single period, but consider it divided equally into $j$ parts flowing at the ends of $j$ equally spaced sub-periods. As well, consider the interest rate $r$ as that assigned to the entire period, but let it be divided by $j$ providing a sub-rate for compounding across the sub-periods. 
The term $C \cdot \mathcal{A}(r, n)$ of Equation (A.1) then becomes

$$
\begin{aligned}
C \cdot \widehat{\mathcal{A}}(r, n): & =\lim _{j \rightarrow \infty} \frac{C}{j} \cdot \frac{1-(1+r / j)^{-j n}}{r / j} \\
& =C \cdot \frac{1-\mathrm{e}^{-r n}}{r}
\end{aligned}
$$

So, if

$$
\widehat{\mathcal{A}}(r, n):=\frac{1-\mathrm{e}^{-r n}}{r},
$$

then Equations (A.1) and (A.2), respectively, become

$$
\widehat{d}_{\mathrm{Mac}}=-\frac{\partial}{\partial r} \log \left[C \cdot \widehat{\mathcal{A}}(r, n)+P \mathrm{e}^{-r n}\right]
$$

and

$$
\widehat{d}_{\text {mod }}=-\frac{\partial}{\partial r} \log \left[C \cdot \widehat{\mathcal{A}}(r, n)+P \mathrm{e}^{-r n}\right],
$$

in the latter case because $\lim _{j \rightarrow \infty}(1+r / j)=1$. So

$$
\widehat{d}_{\text {Mac }}=\widehat{d}_{\text {mod }},
$$

justifying the use of the combined name continuous duration for both versions. As in the case of discrete Macaulay duration, in the simple case of a zero coupon bond continuous duration reduces to the number of periods $n$ to that payment.

An alternative description of this result is that the modified duration is a continuous approximation to the Macaulay duration, or conversely, the Macaulay duration is a discrete approximation to the modified duration. As $n \rightarrow \infty$ with $r n$ constant the two definitions merge.

It is stated without proof that the other common form of annuity timing, payments in advance, i.e., at the beginnings of the compounding periods rather than at the ends, results in the same continuous forms of Equation (A.3).

A.2. Portfolio immunization. An active part of portfolio management is the targeting of a specific duration. For example, a pension fund manager may wish to have a value certain at some future time $t=T$, starting at $t=0$ now. Consider two portfolios $A$ and $B$, with respective durations $d_{A}$ and $d_{B}$, and present values (prices) of $v_{A}$ and $v_{B}$. If these portfolios are combined, then the new portfolio $A+B$ has duration

$$
d_{A+B}=\frac{v_{A}}{v_{A}+v_{B}} d_{A}+\frac{v_{B}}{v_{A}+v_{B}} d_{B}
$$

If $A$ be the portfolio to be immunized to desired duration $d_{A+B}$, then one can solve for $v_{B}$ knowing all other quantities. Specifically,

$$
v_{B}=\frac{d_{A+B}-d_{A}}{d_{B}-d_{A+B}} \cdot v_{A},
$$

which may be positive or negative. If negative one can interpret the result as an amount proportioned to portfolio $B$ to be sold from portfolio $A$ to achieve the objective, or alternatively, the amount to sell short of portfolio $B$. 
Bond immunization is a very big business. In recent years Japanese banking interests have been heavy buyers of 30-year United States Treasury Bond strips - having a duration of 30 years - in order to extend the durations of portfolios. The activity has been so significant as to keep the longest-term yields below those of somewhat shorter-term yields for extended periods of time, even in strongly positive yield curve environments otherwise. 


\section{REFERENCES}

Aihara and Bagchi 2005. Aihara, S. I. and A. Bagchi (2005, Jan.). Stochastic hyperbolic dynamics for infinite-dimensional forward rates and option pricing. Math. Finance 15(1), 27-47.

Bensoussan 1971. Bensoussan, A. (1971). Filtrage optimal des systémes linéaires. In Methodes. Dunod.

Björk, Christensen, and Gombani 1998. Björk, T., B. J. Christensen, and A. Gombani (1998). Some system theoretic aspects of interest rate theory. Insur. Math. Econ. 22, $17-23$.

Björk and Gombani 1999. Björk, T. and A. Gombani (1999). Minimal realizations of interest rate models. Finance Stoch. 3, 413-432.

Björk, Masi, Kabanov, and Runggaldier 1997. Björk, T., G. D. Masi, Y. Kabanov, and W. J. Runggaldier (1997). Towards a general theory of bond markets. Finance Stoch. 1, 141-174.

Black, Derman, and Toy 1990. Black, F., E. Derman, and W. Toy (1990, Jan.-Feb.). A one-factor model of interest rates and its application to Treasury bond options. Financ. Anal. J. 46(1), 33-39.

Borel 1976. Borel, C. (1976). Gaussian random measures on locally convex space. Math. Scand. 38, 265-284.

Brace, Gątarek, and Musiela 1997. Brace, A., D. Ga̧tarek, and M. Musiela (1997, April). The market model of interest rate dynamics. Math. Finance 7(2), 127-155.

Brace and Musiela 1994. Brace, A. and M. Musiela (1994, July). A multifactor Gauss Markov implementation of Heath, Jarrow, and Morton. Math. Finance 4(3), 259-283.

Carmona and Tehranchi 2006. Carmona, R. and M. R. Tehranchi (2006). Interest rate models: an infinite dimensional stochastic analysis perspective. Springer Finance. Berlin: Springer-Verlag. MR2235463 (2008a:91001).

Chatterji and Mandrekar 1978. Chatterji, S. D. and V. S. Mandrekar (1978). Equivalence and singularity of Gaussian measures and applications, Volume 1 of Probabilistic analysis and related topics. New York: Academic Press. MR0478320 (57 \#17804).

Chen 1996a. Chen, L. (1996a). Interest Rate Dynamics, Derivatives Pricing, and Risk Management. Number 435 in Lecture notes in economics and mathematical systems. New York: Springer-Verlag.

Chen 1996b. Chen, L. (1996b). A three-factor model of the term structure of interest rates and its applications in derivatives pricing and risk management. Finan. Markets, Inst. Instruments 5(1), 1-89.

Cox, Ingersoll, Jr., and Ross 1985. Cox, J. C., J. E. Ingersoll, Jr., and S. A. Ross (1985, Mar.). A theory of the term structure of interest rates. Econometrica 53(2), 385-407.

Da Prato and Zabczyk 1992. Da Prato, G. and J. Zabczyk (1992). Stochastic equations in infinite dimensions. Number 44 in Encyclopedia of Mathematics and its Applications. Cambridge: Cambridge University Press. MR1207136 (95g:60073).

Di Nunno, Øksendal, and Proske 2008. Di Nunno, G., B. K. Øksendal, and F. Proske (2008). Malliavin calculus for Lévy processes with applications to finance. Universitext. Berlin: Springer-Verlag.

Filipović 2001. Filipović, D. (2001). Consistency problems for Heath-Jarrow-Morton interest rate models. Number 1760 in Lecture Notes in Mathematics. Berlin: Springer-Verlag. MR1828523 (2002e:91001). 
Filipović and Tappe 2008. Filipović, D. and S. Tappe (2008). Existence of Lévy term structure models. Finance Stoch. 12, 83-115.

Filipović and Zabczyk 2002. Filipović, D. and J. Zabczyk (2002, May). Markovian term structure models in discrete time. Ann. Appl. Probab. 12(2), 710-729.

Gawarecki and Mandrekar 1993. Gawarecki, L. P. and V. S. Mandrekar (1993). Itô-Ramer, Skorohod and Ogawa integrals with respect to Gaussian processes and their interrelationship. In C. Houdré and V. Pérez-Abreu (Eds.), Chaos Expansions, Multiple Wiener-Itô Integrals and Their Applications, Probab. Stochastics Ser., Chapter 18, pp. 349-373. London: CRC Press. First presented to meeting in Guanajuato, Mexico, 27-31 July 1992.

Goldys, Musiela, and Sondermann 2000. Goldys, B., M. Musiela, and D. Sondermann (2000). Lognormality of rates and term structure models. Stoch. Anal. Appl. 18(3), $375-396$.

Haadem and Mandrekar 2010. Haadem, S. and V. S. Mandrekar (2010). A stochastic maximum principle for forward-backward SPDE's.

Heath, Jarrow, and Morton 1992. Heath, D., R. Jarrow, and A. Morton (1992, Jan.). Bond pricing and the term structure of interest rates: a new methodology for contingent claims valuation. Econometrica 60(1), 77-105.

Ho 1992. Ho, T. S. Y. (1992, Sep.). Key rate durations: measures of interest rate risk. $J$. Fixed Income 2(2), 29-44.

Hull and White 1994. Hull, J. C. and A. D. White (1994). The optimal hedging of interest rate sensitive securities. Working paper, Rotman School of Management, University of Toronto.

Jarrow 1978. Jarrow, R. A. (1978, Sep.). The relationship between yield, risk, and return of corporate bonds. J. Finance 33(4), 1235-1240.

Kai 2006. Kai, L. (2006). Stability of infinite dimensional stochastic differential equations with applications. Number 135 in Monographs and Surveys in Pure and Applied Mathematics. Boca Raton: Chapman \& Hall/CRC.

Kunita 1997. Kunita, H. (1997). Stochastic flows and stochastic differential equations. Number 24 in Cambridge Studies in Advanced Mathematics. Cambridge: Cambridge University Press. MR1472487 (98e:60096) Reprint of the 1990 original.

Lee and Ho 1986. Lee, S.-B. and T. S. Y. Ho (1986). Term structure movements and pricing interest rate contingent claims. J. Finance 41(5), 1011-1029.

Ma and Yong 1999. Ma, J. and J. Yong (1999). Forward-backward stochastic differential equations and their applications. Number 1702 in Lecture Notes in Mathematics. Berlin: Springer.

Macaulay 1938. Macaulay, F. R. (1938). Some theoretical problems suggested by the movements of interest rates, bond yields and stock prices in the United States since 1856. New York: Columbia University Press.

Mandrekar and Zhang 1993. Mandrekar, V. S. and S. Zhang (1993). Skorokhod integral and differentiation for Gaussian processes. In J. Ghosh, S. Mitra, K. Parthasarathy, and B. P. Rao (Eds.), Statistics \&5 Probability: Festschrift in honor of Raghu Raj Bahadur, pp. 395-410. New Delhi: John Wiley Eastern.

Musiela 1995. Musiela, M. (1995). General framework for pricing derivative securities. Stochastic Process. Appl. 55, 227-251.

Musiela and Rutkowski 1997. Musiela, M. and M. Rutkowski (1997). Continuous-time term structure models: forward measure approach. Finance Stoch. 1, 261-291. 
Nakayama 2002. Nakayama, T. (2002). Approximation of BSDE's by stochastic difference equations. J. Math. Sci. Univ. Tokyo 9, 257-277.

Nualart 1995. Nualart, D. (1995). The Malliavin calculus and related topics. Probability and its applications. Berlin: Springer-Verlag.

Øksendal, Proske, and Zhang 2005. Øksendal, B. K., F. Proske, and T. Zhang (2005). Backward stochastic partial differential equations with jumps and application to optimal control of random jump fields. Stochastics 77(5), 381-399.

Peng 1997. Peng, S. (1997). Backward SDE and related $g$-expectation. In N. El Karoui and L. Mazliak (Eds.), Backward Stochastic Differential Equations, Number 364 in Pitman Research Notes in Mathematics. Berlin: Springer-Verlag.

Prévôt and Röckner 2007. Prévôt, C. and M. Röckner (2007). A concise course on stochastic partial differential equations. Number 1905 in Lecture Notes in Mathematics. Berlin: Springer-Verlag. MR2329435 (2009a:60069).

Rendleman and Bartter 1980. Rendleman, Jr., R. J. and B. J. Bartter (1980, Mar.). The pricing of options on debt securities. J. Finan. Quant. Anal. 15(1), 11-24.

Ritchken and Sankarasubramanian 1995. Ritchken, P. H. and L. Sankarasubramanian (1995, Jan.). Volatility structures of forward rates and the dynamics of the term structure. Math. Finance 5(1), 55-72.

Üstünel 1995. Üstünel, A. S. (1995). An introduction to analysis on Wiener space. Number 1610 in Lecture Notes in Mathematics. Berlin: Springer-Verlag. MR1439752 (98d:60109).

Vargiolu 1999. Vargiolu, T. (1999). Invariant measures for the Musiela equation with deterministic diffusion term. Finance Stoch. 3, 483-492.

Vašíček 1977. Vašíček, O. A. (1977). An equilibrium characterization of the term structure. J. Finan. Econ. 5, 177-188.

Zhang 2004. Zhang, J. (2004). A numerical scheme for BSDEs. Ann. Appl. Probab. 14(1), 459-488. MR2023027 (2004j:65015). 
(Paul C. Kettler)

Centre of Mathematics for Applications Department of Mathematics

UNIVERSITY OF OSLO

P.O. Box 1053, Blindern

$\mathrm{N}-0316$ OSLO

NORWAY

E-mail address: paulck@math.uio.no

$U R L:$ http://www . math.uio.no/ paulck/

(Frank Proske)

Centre of Mathematics for Applications

Department of Mathematics

UNIVERSITY OF OSLO

P.O. Box 1053, BLINDERN

$\mathrm{N}-0316$ OSLO

NORWAY

E-mail address: proske@math.uio.no

$U R L:$ http://www.math.uio.no/

(Mark Rubtsov)

Centre of Mathematics for Applications

Department of Mathematics

UNIVERSITY OF OSLO

P.O. Box 1053, Blindern

$\mathrm{N}-0316$ OSLO

NORWAY

E-mail address: mark.rubtsov@cma.uio.no

$U R L:$ http://www.math.uio.no/ 